\title{
TURBULENCE APPEARANCE AT THE BOTTOM OF A SOLITARY WAVE
}

\author{
Paolo Blondeaux ${ }^{1}$, Jan O. Pralits ${ }^{1}$, Giovanna Vittori ${ }^{1}$
}

\begin{abstract}
The conditions leading to transition and turbulence appearance at the bottom of a solitary waveare determined by means of a linear stability analysis of the laminar flow in the bottom boundary layer. The ratio between the wave amplitude and the thickness of the viscous bottom boundary layer is assumed to be large and a 'momentary' criterion of instability is used. The results obtained show that the laminar regime becomes unstable, during the decelerating phase, if the height of the wave is larger than a threshold value which depends on the ratio between the boundary layer thickness and the local water depth. A comparison of the theoretical results with the experimental measurements of Sumer et al. (2010) seems to support the stability analysis.
\end{abstract}

Keywords: boundary layer stability, coastal engineering, solitary waves

\section{INTRODUCTION}

Even though in nature it is not easy to observe a truly solitary wave (Shore Protection Manual, 1984), the solitary wave model provides a fair description of sea waves when they approach the nearshore region (Munk, 1949). Moreover, the solitary wave model approximate the long waves generated by earthquakes and landslides (tsunami). Therefore, a lot of studies have been made of the flow generated by the propagation of a solitary wave. The Reynolds number is usually quite large and it is possible to split the fluid domain into an inviscid region and viscous boundary layers. A review of the analyses of the flow in the inviscid region can be found in Miles (1980). Even if the bottom boundary layer is quite thin, the description of the flow close to the bottom is quite important since the propagation of a solitary wave can mobilize large amounts of sediment and give rise to significant erosion and deposition processes. In the recent years, many studies have been made of the boundary layer at the bottom of a solitary wave but many of them consider the laminar regime. For example, Liu \& Orfilia (2004) introduced the Boussinesq approximation and derived depth integrated continuity and momentum equations for transient long waves. The resulting equations are differential-integral equations, in terms of the depth-averaged horizontal velocity and the free surface displacement, in which viscous effects are taken into account by convolution integrals. Then, Liu et al. (2007), using Liu \& Orfilia's (2004) solution, examined the boundary layer under a solitary wave and found that the velocity inside the bottom boundary layer reverses its direction during the decelerating phase, even though the free-stream velocity always points in the direction of wave propagation.

The velocity field in the turbulent boundary layer under a solitary wave has been recently obtained by Vittori \& Blondeaux $(2008,2011)$ by means of direct numerical simulations. Vittori \& Blondeaux $(2008$, 2011) assumed that turbulence structure is unaffected by the 'slow' spatial variations of the inviscid external flow and used a 'local' model. Their results show that the flow regime is laminar if the wave height is relatively small. Turbulence starts to appear if the wave height exceeds a threshold value which depends on the ratio between the boundary layer thickness and the water depth. Moreover, the numerical simulations show that, close to the critical conditions, turbulence appears only during the decelerating phase. Turbulence is generated also during the accelerating only the the wave height is much larger that its critical value. The numerical results have also shown that also in the turbulent regime, the velocity reverses its direction and points offshore during the late decelerating phase.

The recent laboratory measurements of Sumer et al. (2010) have shown that the transition between the laminar regime and the turbulent regime depends on the value of the Reynolds number $R e$ defined by Sumer et al. (2010) as $R e=4 H^{3 / 2} /\left(\sqrt{3} \delta^{2}\right)$, where $H$ is the ratio between the wave height $H^{*}$ and the water depth $h^{*}$ and $\delta$ is the ratio between the boundary layer thickness $\delta^{*}$ and $h^{*}$. Indeed, when the Reynolds number is smaller than $2 \times 10^{5}$, the velocity field does not deviate from the laminar solution described by Liu et al.

\footnotetext{
${ }^{1}$ DICCA, University of Genoa, Via Montallegro 1, 16122, Genoa, Italy
} 
(2007). However, for values of Re slightly larger tha $2 \times 10^{5}$, the values of the bed shear stress measured by Sumer et al. (2010) show large peaks which are related to the appearance of quasi-two-dimensional vortex tubes. Moreover, an increase of the Reynolds number above $5 \times 10^{5}$ leads to the appearance of multiple irregular spikes in the measured values of the bed shear stress which are originated by turbulence appearance.

In the present work, we consider the bottom boundary layer generated by the propagation of a solitary wave and we brielfy summarize the linear stability analysis of the laminar regime made by Blondeaux et al. (2012). The analysis introduces a 'momentary' criterion for instability as that used by Shen (1961) and by Blondeaux \& Seminara (1979) to investigate the stability of the oscillatory bottom boundary layer generated by monochromatic linear surface waves. In particular, the transition is assumed to take place for large values of the Reynolds number such to assume that the growth of the perturbations takes place on a time scale much faster than the time scale of the basic flow. A normal mode decomposition is applied and the analysis is carried out by considering the generic Fourier component of the perturbation. Finally, taking into account the results of Conrad \& Criminale (1965), the analysis considers only two-dimensional perturbations which are more unstable than three-dimensional perturbations. The results explain the appearance of the vortex tubes observed by Sumer et al. (2010) and predict their spacing, even though the critical value of the Reynolds number is underestimated. A justification of the latter finding is provided in the discussion of the results.

\section{THE BASIC FLOW}

The propagation of a two-dimensional solitary wave of height $H^{*}$ is considered. The water depth $h^{*}$ is assumed to be constant. A Cartesian coordinate system $\left(X_{1}^{*}, X_{2}^{*}, X_{3}^{*}\right)$ is introduced, having the $\left(X_{1}^{*}, X_{3}^{*}\right)$-plane coincident with the bottom, the $X_{1}^{*}$-axis in the direction of wave propagation and the $X_{2}^{*}$-axis pointing in the up-ward direction (a star denotes dimensional quantities). As pointed out in the introduction, the Reynolds number of the phenomenon turns out to be large and the flow field can be split into i) a core region, where the velocity field is irrotational and the fluid behaves like an inviscid fluid, and ii) two boundary layers, where viscous effects are important. The description of the flow into the inviscid region can be obtained by assuming that the height $H^{*}$ of the wave is much smaller than the local water depth $h^{*}$ and that the latter is much smaller than the length $L^{*}$ of the wave. In particular the ratio $H=H^{*} / h^{*}$ is assumed be of the same order of magnitude as $\mu^{2}=h^{*} / L^{*}$ (Boussinesq approximation). If terms of order $H^{2}$ are neglected, the free surface elevation $\eta^{*}$ with respect to the still water level and the horizontal velocity component induced by the propagation of the solitary wave were obtained by Grimshaw (1971) and are

$$
\begin{gathered}
\eta^{*}\left(X_{1}^{*}, t^{*}\right)=H^{*} \operatorname{sech}^{2}\left(\sqrt{\frac{3 H}{4}} \frac{X_{1}^{*}-\sqrt{g^{*} h^{*}} t^{*}}{h^{*}}\right) \\
V_{1}^{*}\left(X_{1}^{*}, t^{*}\right)=H \sqrt{g^{*} h^{*}} \operatorname{sech}^{2}\left(\sqrt{\frac{3 H}{4}} \frac{X_{1}^{*}-\sqrt{g^{*} h^{*}} t^{*}}{h^{*}}\right),
\end{gathered}
$$

The vertical velocity component is much smaller than the horizontal component and turns out to be

$V_{2}^{*}\left(X_{1}^{*}, X_{2}^{*}, t^{*}\right)=-H^{3 / 2} \sqrt{3 g^{*} h^{*}}\left(\frac{X_{2}^{*}}{h^{*}}\right) \operatorname{sech}^{2}\left(\sqrt{\frac{3 H}{4}} \frac{X_{1}^{*}-\sqrt{g^{*} h^{*} t^{*}}}{h^{*}}\right) \tanh \left(\sqrt{\frac{3 H}{4}} \frac{X_{1}^{*}-\sqrt{g^{*} h^{*}} t^{*}}{h^{*}}\right)$

In the following, the surface boundary layer is not considered, because it is much weaker than that located close to the bottom.

The solution in the inviscid region suggests that $\frac{\partial}{\partial t^{*}} \sim \frac{\sqrt{g^{*} h^{*}}}{h^{*}}$ while $\nu^{*} \frac{\partial^{2}}{\partial x_{2}^{* 2}} \sim \frac{\nu^{*}}{\delta^{*}}$, where $\nu^{*}$ is the kinematic viscosity of the sea water (hereinafter the scaling introduced by Grimwshaw (1971) is used since 
it avoids the appearance of the parameter $\mu$ into the problem). Hence, the boundary layer thickness $\delta^{*}$ turns out to be $O\left(\sqrt{\frac{\nu^{*} h^{*}}{\sqrt{g^{*} h^{*}}}}\right)$ which is much smaller than $h^{*}$. Moreover, an order of magnitude analysis suggests that i) the vertical velocity component is negligible, ii) the pressure is independent of the vertical coordinate, iii) the horizontal velocity component $v_{b 1}^{*}$ in the boundary layer, which depends only parametrically on the coordinate $X_{1}^{*}$, can be obtained by solving

$$
\frac{\partial v_{b 1}^{*}}{\partial t^{*}}=\left.\frac{\partial V_{1}^{*}}{\partial t^{*}}\right|_{X_{2}=0}+\nu^{*} \frac{\partial^{2} v_{b 1}^{*}}{\partial X_{2}^{* 2}}
$$

where the streamwise pressure gradient term has been replaced by $\partial V_{1}^{*} /\left.\partial t^{*}\right|_{X_{2}^{*}=0}$. Of course, the flow field described by (4) should match the irrotational flow outside the boundary layer and satisfy the no-slip condition at the bottom. The solution can be found following the procedure described by Mei (1989) and reads

$$
\begin{aligned}
& v_{b 1}^{*}\left(X_{1}^{*}, X_{2}^{*}, t^{*}\right)=V_{1}^{*}\left(X_{1}^{*}, t^{*}\right) \\
& \quad-H \sqrt{g^{*} h^{*}} \frac{2}{\sqrt{\pi}} \int_{0}^{\infty} \operatorname{sech}^{2}\left[\sqrt{\frac{3 H}{4}}\left(\frac{X_{2}^{* 2} \sqrt{g^{*} h^{*}}}{4 \nu^{*} h^{*} \xi^{2}}+\frac{X_{1}^{*}-\sqrt{g^{*} h^{*}} t^{*}}{h^{*}}\right)\right] e^{-\xi^{2}} d \xi
\end{aligned}
$$

\section{THE STABILITY ANALYSIS}

Since, the aim of the analysis is the study of the stability of the flow in the bottom boundary layer, let us introduce dimensionless spatial and temporal coordinates $\left(x_{1}, x_{2}, x_{3}\right), t$ defined by

$$
\left(x_{1}, x_{2}, x_{3}\right)=\frac{\left(X_{1}^{*}, X_{2}^{*}, X_{3}^{*}\right)}{\delta^{*}}, \quad t=\frac{t^{*} \sqrt{g^{*} h^{*}}}{h^{*}}
$$

where $\delta^{*}$ is given by

$$
\delta^{*}=\sqrt{\frac{2 \nu^{*} h^{*}}{\sqrt{g^{*} h^{*}}}}
$$

Moreover, let us use dimensionless velocity components $\left(v_{1}, v_{2}, v_{3}\right)$ and dimensionless pressure field $p$ defined by

$$
\left(v_{1}, v_{2}, v_{3}\right)=\frac{\left(v_{1}^{*}, v_{2}^{*}, v_{3}^{*}\right)}{H \sqrt{g^{*} h^{*}}}, \quad p=\frac{p^{*}}{\rho^{*} H g^{*} \delta^{*}}
$$

$\rho^{*}$ being the density of the sea water.

Because of Squire's theorem, let us consider two-dimensional perturbations of the flow field described by (5). The velocity and the pressure are thus given by

$$
\left(v_{1}, v_{2}, p\right)=\left(v_{b 1}, 0, p_{b}\right)+\epsilon\left(v_{p 1}, v_{p 2}, p_{p}\right)
$$

where the dimensionless velocity $v_{b 1}=v_{b 1}^{*} /\left(H \sqrt{g^{*} h^{*}}\right)$ of the basic flow is provided by (5).

If the amplitude $\epsilon$ of the perturbation is assumed to be much smaller than one, it is possible to study the time development of the perturbations by neglecting nonlinear terms and to consider the following equations which are obtained by continuity and momentum equations.

$$
\begin{gathered}
\frac{\partial v_{p 1}}{\partial x_{1}}+\frac{\partial v_{p 2}}{\partial x_{2}}=0 \\
\frac{\partial v_{p 1}}{\partial t}+\frac{H}{\delta}\left[v_{b 1} \frac{\partial v_{p 1}}{\partial x_{1}}+v_{p 2} \frac{\partial v_{b 1}}{\partial x_{2}}\right]=-\frac{\partial p_{p}}{\partial x_{1}}+\frac{1}{2}\left[\frac{\partial^{2} v_{p 1}}{\partial x_{1}^{2}}+\frac{\partial^{2} v_{p 1}}{\partial x_{2}^{2}}\right]
\end{gathered}
$$




$$
\frac{\partial v_{p 2}}{\partial t}+\frac{H}{\delta}\left[v_{b 1} \frac{\partial v_{p 2}}{\partial x_{1}}\right]=-\frac{\partial p_{p}}{\partial x_{2}}+\frac{1}{2}\left[\frac{\partial^{2} v_{p 2}}{\partial x_{1}^{2}}+\frac{\partial^{2} v_{p 2}}{\partial x_{2}^{2}}\right]
$$

where the dimensionless parameter $\delta$

$$
\delta=\frac{\delta^{*}}{h^{*}}
$$

is introduced. Mass conservation can be forced by introducing a stream function $\psi$ such that

$$
\left(v_{p 1}, v_{p 2}\right)=\left(\frac{\partial \psi}{\partial x_{2}},-\frac{\partial \psi}{\partial x_{1}}\right)
$$

Then, eliminating the pressure field from (11) and (12), the linearized vorticity equation is obtained

$$
\frac{\partial^{3} \psi}{\partial t \partial x_{1}^{2}}+\frac{\partial^{3} \psi}{\partial t \partial x_{2}^{2}}+\frac{H}{\delta}\left[v_{b 1}\left(\frac{\partial^{3} \psi}{\partial x_{1}^{3}}+\frac{\partial^{3} \psi}{\partial x_{2}^{2} \partial x_{1}}\right)-\frac{\partial^{2} v_{b 1}}{\partial x_{2}^{2}} \frac{\partial \psi}{\partial x_{1}}\right]=\frac{1}{2}\left(\frac{\partial^{4} \psi}{\partial x_{1}^{4}}+2 \frac{\partial^{4} \psi}{\partial x_{1}^{2} \partial x_{2}^{2}}+\frac{\partial^{4} \psi}{\partial x_{2}^{4}}\right)
$$

Since the perturbations are assumed of small amplitude and the problem has been linearized, a normal mode approach is used. Hence, the generic spatial component along the $x_{1}$-axis is considered. Taking into account that, for field conditions, the thickness $\delta^{*}$ of the viscous boundary layer is much smaller than the height $H^{*}$ of the wave, the value of the ratio $H / \delta$ appearing in (15), turns out to be much larger than one. As pointed out by Blondeaux et al. (2012), simple algebra shows that the ratio $H / \delta$ is equal to the ratio between the characteristic temporal scale of the wave motion $h^{*} / \sqrt{g^{*} h^{*}}$ (see 6) and the convective temporal scale characteristic of the time development of the perturbation $\delta^{*} /\left(H \sqrt{g^{*} h^{*}}\right)$ (the latter is assumed to be equal to the ratio between the thickness of the bottom boundary layer and the scale of the fluid velocity (see 8 )). Therefore, the amplitude of the perturbation can be assumed to grow on a time scale much faster than that which characterizes the time development of the basic flow and a 'momentary' criterion for instability can be used (see Shen (1961) and Blondeaux \& Seminara (1979)). It follows that the stream function appearing in (15) can be written in the form

$$
\psi\left(x_{1}, x_{2}, t\right)=\operatorname{Real}\left\{f\left(x_{2}, t\right) \exp \left[i \alpha\left(x_{1}-\frac{H}{\delta} \int c(\tau) d \tau\right)\right]\right\}+\text { h.o.t. }
$$

where $\alpha$ is the streamwise wavenumber of the generic Fourier component of the perturbation, the real part $\left(c_{r}\right)$ of $c$ is the wave speed and the imaginary part $\left(c_{i}\right)$ provides the growth/decay of the perturbation. Moreover, h.o.t. indicates higher order terms.

An ordinary differential equation for $f$ can be obtained by substituting (16) into (15)

$$
\left[v_{b 1}\left(x_{2}, t\right)-c(t)\right] N^{2} f\left(x_{2}, t\right)-\frac{\partial^{2} v_{b 1}\left(x_{2}, t\right)}{\partial x_{2}^{2}} f\left(x_{2}, t\right)=\frac{1}{2 i \alpha(H / \delta)} N^{4} f\left(x_{2}, t\right)
$$

where $t$ is just a parameter and the operator $N^{2}$ is defined by

$$
N^{2}=\frac{\partial^{2}}{\partial x_{2}^{2}}-\alpha^{2}
$$

The problem is closed by the homogeneous boundary conditions at the bottom and far from it. Indeed, the perturbation of the velocity should vanish both at the wall and far from it. The viscous term, which is of order $1 /(H / \delta)$, is retained in (17), since it cannot be neglected within a viscous layer close to the wall and possible critical layers. As discussed in Blondeaux et al. (2012), a formal approach would require the solution of the inviscid version of (17) and the matching of the inviscid solution with the solution in the viscous and critical layers. However, this procedure involves a lot of heavy algebra. Since, Blondeaux \& Vittori (1994) showed that the direct solution of the equation (17) provides the same results of the more rigorous approach, the direct solution has been preferred. 




Figure 1. Dimensionless growth rate $c_{i}$ plotted versus the wave phase $\theta$ for $h^{*}=1.87 \mathbf{m}$ and $H^{*}=0.22 \mathbf{m}$ considering an harmonic component of the perturbation characterized by a wavelength $\lambda^{*}$ equal to $2.94 \mathbf{c m}$.

We note that the time $t^{*}$ and the coordinate $X_{1}^{*}$ appear into the problem only in the combination $\theta=$ $X_{1}-t$, which can be considered as the phase of the solitary wave and a parameter of the problem. Hence, equation (17) is solved for different values of $\theta$ which correspond to different locations and/or to different phases within the wave cycle. In the discussion of the results, the location characterized by $X_{1}=0$ is considered such that a vanishing value of $\theta$ corresponds to the passage of the wave crest.

A numerical approach is used to solve the problem. A compact finite difference approach of fourth-order is used to discretize equation (17). The matching with the inviscid solution is forced at $x_{2}=30$. To find a non-trivial solution, it is necessary to force an eigenrelation which provides the value of $c$ as function of $\alpha$ and the other parameters of the problem. The least stable eigenvalue is obtained by inverse iteration.

\section{DISCUSSION OF THE RESULTS}

Even though the problem is formulated in dimensionless form and the solution is obtained using dimensionless variable, the results are discussed using physical dimensional variables to allow the practical relevance of the results to be evaluated. If the kinematic viscosity of sea water is considered and $\nu^{*}$ is fixed equal to $1.0 \times 10^{-6}$, the stability problem previously formulated depends on the water depth $h^{*}$, the wave height $H^{*}$ and the dimensionless parameter $\theta$ which is related to the phase of the solitary wave. In the following, the eigenrelation and the eigensolution are determined for fixed values of $h^{*}$ and $H^{*}$ and by varying the phase $\theta$ within the wave cycle. Since the value of the coordinate $X_{1}^{*}$ is considered fixed and equal to zero, values of $\theta$ smaller than zero correspond to phases before the passage of the wave crest while values of $\theta$ greater than zero to phases after the passage of the crest.

Figure 1 shows the phase dependence of the dimensionless growth rate $c_{i}$ of the harmonic component characterized by a wavelength $\lambda^{*}=2.94 \mathrm{~cm}$, for $h^{*}=1.87 \mathrm{~m}$ and $H^{*}=0.22 \mathrm{~m}$. The values of the parameters $h^{*}$ and $H^{*}$ are those describing the experiment of Sumer et al. (2010) characterized by a maximum value of the free stream velocity $U_{0 m}^{*}=50.9 \mathrm{~cm} / \mathrm{s}$ and $T^{*}=4 \pi h^{*} / \sqrt{3 g^{*} H^{*}}=9.3 \mathrm{~s}(R e=$ $\left.3.8 \times 10^{5}\right)$. The value $\lambda^{*}=2.94 \mathrm{~cm}(\alpha=0.2)$ has been chosen because it is the critical wavelength, i.e. it corresponds to the harmonic component which is first destabilized. During the accelerating phase, the value of $c_{i}$ is negative and the harmonic component characterized by $\lambda^{*}=2.94 \mathrm{~cm}$ tends to decay. The growth rate $c_{i}$ keeps negative during the early decelerating phase, thus showing that the harmonic 




Figure 2. Dimensionless value of $c_{r}$ plotted versus the wave phase $\theta$ for $h^{*}=1.87 \mathbf{m}$ and $H^{*}=0.22 \mathbf{m}$ considering an harmonic component of the perturbation characterized by a wavelength $\lambda^{*}$ equal to $2.94 \mathbf{c m}$.

component is stable also after the passage of the wave crest. However, as soon as the dimensionless wave phase $\theta$ becomes larger than about 1.0, the growth rate becomes positive and the harmonic component characterized by a wavelength equal to $3.9 \mathrm{~mm}$ tends to grow. Figure 2 shows the value of $c_{r}$, which is a quantity strictly related to the migration speed of the perturbation component. Up to about $\theta=4.4$, the value $c_{r}$ is positive, thus indicating that the perturbation component migrates in the direction of wave propagation. Only, for values of $\theta$ larger than $4.4, c_{r}$ becomes negative showing that the perturbation component reverses its velocity at the late decelerating phase because of flow reversal within the boundary layer.

Similar results are obtained for different values of $\lambda^{*}$ and the stability/instability of the flow within the bottom boundary layer can be analysed by looking at figure 3 , which shows the value of $c_{i}$ as function of the wave phase $\theta$ and $\lambda^{*}$ for the same values of $h^{*}$ and $H^{*}$ as those considered in figures 1 and 2. In the plane $\left(\theta, \lambda^{*}\right)$ a region can be observed where the value of $c_{i}$ is positive, thus indicating that i) the laminar regime is unstable, ii) the instability takes place during the decelerating phase, iii) a two-dimensional wave is generated by the instability of the basic unidirectional flow. Indeed, if the fastest growing mode (f.g.m.) is assumed to prevail on the other modes, the linear analysis predicts the appearance of a periodic pattern characterized by a wavelength $\hat{\lambda}^{*}$ which gives rise to the maximum growth rate for a given value of $\theta$. Moreover, a periodic pattern is predicted to appear for $\theta>\theta_{c}$, i.e. as soon as $c_{i}$ becomes positive. Because of the slow temporal variation of the basic flow, the wavelength $\hat{\lambda}^{*}$ of the most unstable harmonic component depends on the phase within the wave cycle. Hence the periodic pattern, the appearance of which is predicted by the analysis, would be characterized by a wavelength which depends on time. However, as pointed out by Blondeaux et al. (2012), the wavelength of the perturbation cannot change continuously but only through the appearance of defects. Unfortunately, the growth of these defects cannot be determined by using a linear approach. Therefore, in the following, the wavelength of the periodic patterns which are expected to appear is assumed to be coincident with that predicted for the critical value of the paramenter $\theta$, i.e. at $\theta=\theta_{c}$. This procedure is supported by the analysis of the movies of Sumer et al. (2010) which can be seen at http://journals.cambridge.org in the supplementary material of the paper. In fact, the experimental results of Sumer et al. (2010) do not show any significant change of the distance between adjacent vortex tubes as they develop. The results of figure 3 show that $\theta_{c}$ is equal to about 1.0 and $\hat{\lambda}^{*}$ evaluated at $\theta=\theta_{c}$ is equal to about $2.94 \mathrm{~cm}$. The reliability of the analysis has been ascertained by comparing the theoretical 




Figure 3. Growth rate $c_{i}$ ( continuous line, $c_{i}>0$; continuous thick line, $c_{i}=0 ; \Delta c_{i}=0.005$ ) plotted versus $\theta$ and $\lambda^{*}$ for $h^{*}=1.87 \mathbf{m}$ and $H^{*}=0.22 \mathbf{m}$.

findings with the experimental measurements of Sumer et al. (2010). Indeed the movies of Sumer et al. (2010) allow an estimate of the wavelength of the fastest growing mode to be obtained, if the growth of the latter is assumed to give rise to the vortex tubes observed in the experiments. For $U_{0 m}^{*}=50.9 \mathrm{~cm} / \mathrm{s}$ and $T^{*}=9.3 \mathrm{~s}$, the average distance between the adjacent vortex tubes recorded by Sumer et al. (2010) is about $2.3 \mathrm{~cm}$, a value which is close to the value predicted by the analysis. Moreover, a periodic pattern is observed $1.53 \mathrm{~s}$ after the free stream velocity attains its maximum, i.e. at $\theta=3.18$. A fair agreement is found between the theoretical results and the experimental data as far as the wavelength of fastest growing mode is considered. On the other hand, the value of $\theta$ at which the periodic patterns are predicted to appear is significantly underestimaed. This finding can be explained considering that the perturbation takes some time to attain an amplitude large enough to be observed. The theoretical predictions are compared with all the flow visualizations which are available at http://journals.cambridge.org and with a further movie which has been made available by the experimentalists. As discussed by Blondeaux et al. (2012), results similar to the previous ones are found for all the experiments available at http://journals.cambridge.org in the supplementary material of the paper and for a further experiments kindly provided by Sumer and cowokers.

The results of Vittori \& Blondeaux $(2008,2011)$, which are obtained by means of direct numerical simulations of Navier-Stokes and continuity equations, show that turbulence appearance is observed when the ratio $H$ between $H^{*}$ and $h^{*}$ is larger than a critical value $H_{c}$, which depends on the ratio $\delta=\delta^{*} / h^{*}$. This behaviour of $H_{c}(\delta)$ was observed also in the experiments of Sumer et al. (2010). Indeed, Sumer et al. (2010) defined the Reynolds number as $R e=4 H^{3 / 2} /\left(\sqrt{3} \delta^{2}\right)$ and observed vorted tubes when $R e$ is larger than $2 \times 10^{5}$. Even though the qualitative behaviour of the results of the present stability analysis agrees with that found by Vittori \& Blondeaux (2008) and Sumer et al. (2010), significant quantitative differences are found. Indeed, the present stability analysis indicates instability of the laminar regime for values of $H$ smaller than those predicted numerically and/or observed experimentally. For example, for $h^{*}=0.58 \mathrm{~m}$, the theoretical results show that the critical value of $H^{*}$ falls around $1.5 \mathrm{~cm}$ (see figure 4), a value which is much smaller than that proposed by Sumer et al. (2010) and predicted numerically by Vittori \& Blondeaux (2008). This finding can be understood looking at figure 5, which shows results obtained by means of direct numerical simulations made with the same code used by Vittori \& Blondeaux (2008). In particular, figure 5 

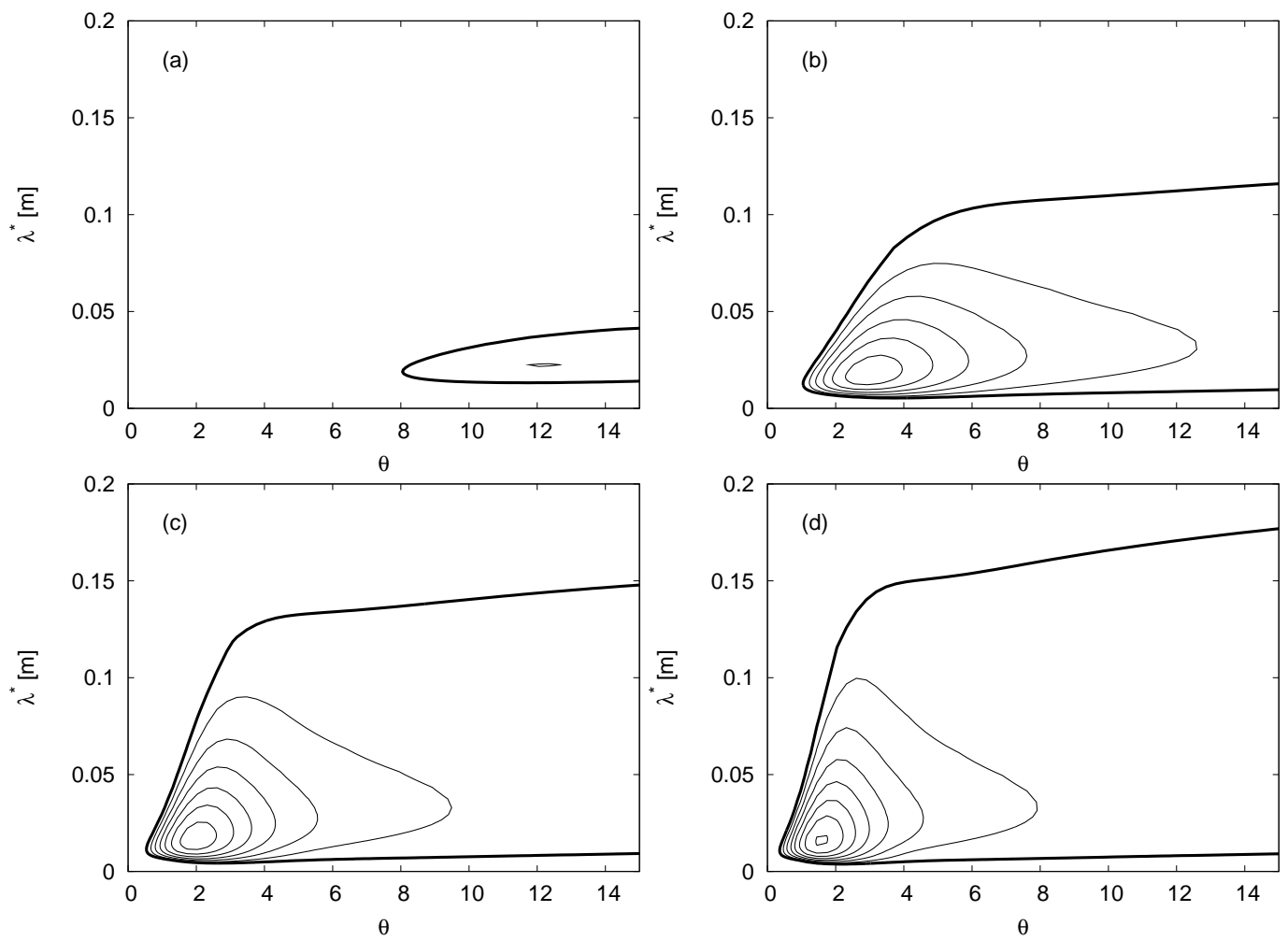

Figure 4. Growth rate $c_{i}$ (continuous line, $c_{i}>0$; continuous thick line $c_{i}=0 ; \Delta c_{i}=0.01$ ) plotted versus $\theta$ and $\lambda^{*}$ for $h^{*}=0.58 \mathbf{m}$ and a) $\left.\left.H^{*}=1.5 \mathbf{c m ~ b ) ~} H^{*}=11.6 \mathbf{c m}, \mathbf{c}\right) H^{*}=23.2 \mathbf{c m}, \mathbf{d}\right) H^{*}=34.8 \mathbf{c m}$.

shows the dimensionless kinetic energy per unit area $K$ of the flow perturbations plotted versus the phase $\theta$ within the wave cycle for $h^{*}=0.58 \mathrm{~m}$ and different values of $H^{*}$.

For $H^{*}=1.5 \mathrm{~cm}$ the value of $K$ is negligible during the whole wave cycle as predicted by the present linear stability analysis (see figure 4 a). If $H^{*}$ is slightly increased, $K$ grows during the late decelerating phase as predicted by the present linear stability analysis. However up to $H^{*}=23.2 \mathrm{~cm}$, the growth of $K$ is weak and does not lead to the appearance of detectable perturbations of the velocity field which is practically coincident with that predicted by (5). Only when $H$ is equal to $29 \mathrm{~cm}$ or larger, the strength attained by the perturbations is significant and the flow deviates from the laminar flow. Of course, the process is continuous and there is some arbitrariness in defining the value of $H^{*}$ which gives rise to significant perturbations. Indeed both Vittori \& Blondeaux $(2008,2011)$ introduced an arbitrary threshold value of $K$ to discriminate between the laminar and the turbulent regimes. The results plotted in figure 5 explain also the differences between the values of $\theta_{c}$ predicted by the present stability analysis and those at which Sumer et al. (2010) observed vortex tubes. Indeed, figure 5 shows that there is a significant time delay between the beginning of the growth of the perturbations and the phase at which they attain a significant level. When comparing the present theoretical results with the numerical finding of Vittori \& Blondeaux (2008) and the experimental measurements of Sumer et al. (2010), the reader should consider that for small values of $H^{*}$ the stability analysis is not expected to provide accurate quantitative results because the ratio $H^{*} / \delta^{*}$ is not large enough in order to reliably apply a "momentary" criterion for instability.

\section{CONCLUSIONS}

The stability analysis previously described and the results it provides show that the laminar boundary layer at the bottom of a solitary wave, propagating over a constant water depth, is unstable if the height 


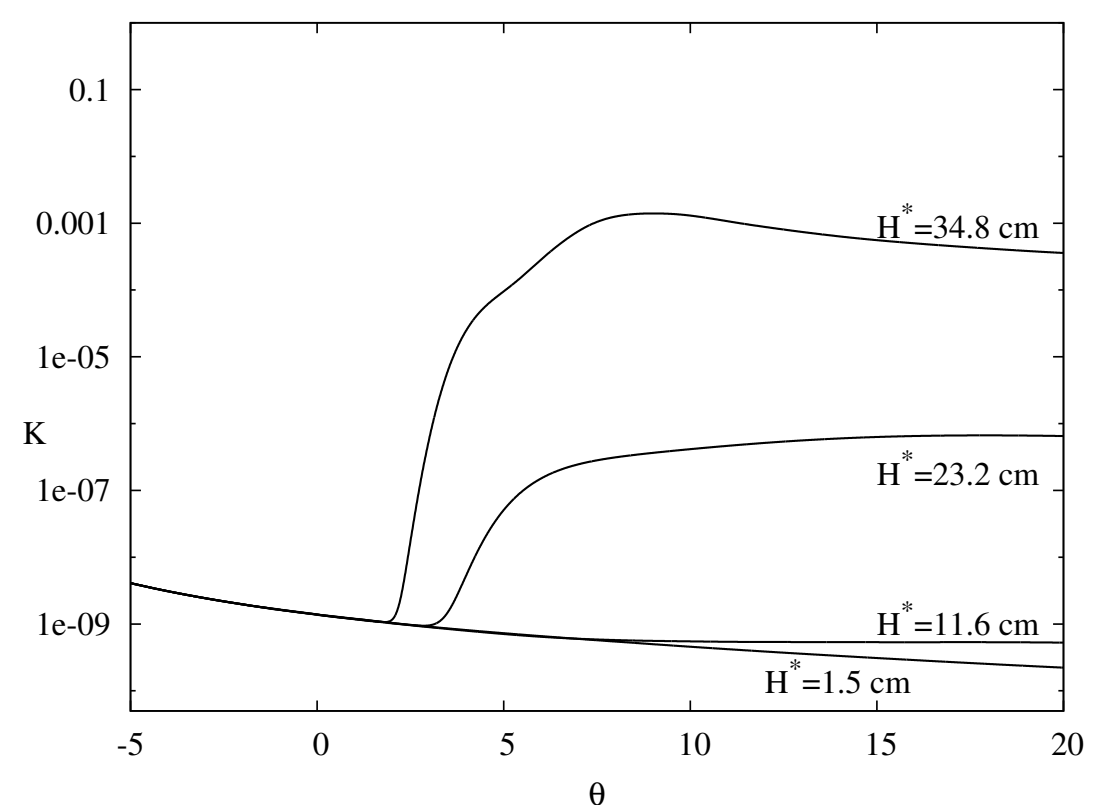

Figure 5. Dimensionless kinetic energy $K$ of flow perturbations plotted versus $\theta$ for $h^{*}=0.58 \mathbf{m}$ and different values of $H^{*}$.

of the wave exceeds a threshold value which depends on the thickness of the boundary layer. In the range of the parameters presently investigated, the instability takes place during the decelerating phase of the wave cycle. The wavelength of the fastest growing mode, at the critical conditions, is found to be similar to the distance between the adjacent vortex tubes observed experimentally by Sumer et al. (2010). A comparison of the theoretical results with the numerical findings of Vittori \& Blondeaux (2008) and the experimental visualizations of Sumer et al. (2010) seems to indicate that the stability analysis underpredicts the threshold value of the wave height triggering the instability of the laminar regime. However, a careful analysis of the results shows that the discrepancy between the predictions of the linear stability analysis and the numerical/experimental results can be explained by considering the unsteady character of the basic flow which does not allow a significant growth of the perturbation to be attained during the early stages of the instability.

\section{ACKNOWLEDGEMENTS}

This study has been funded by the 'Ministero dell'Istruzione, dell'Universitá e della Ricerca' under the research project 2008YNPNT9-003 'Idrodinamica e morfodinamica nella regione dei frangenti'. The authors thank Prof. Sumer and Dr. Carstensen who made available a movie of the experiments and measurements of the characteristics of the vortex tubes.

\section{REFERENCES}

Blondeaux, P. 1987 Turbulent boundary layer at the bottom of gravity waves. J. Hydraulic Res., 25 (4), 447-464.

Blondeaux, P., Pralits J. \& Vittori, G. 2012 Transition to turbulence at the bottom of a solitary wave J. Fluid Mech. DOI: http://dx.doi.org/10.1017/jfm.2012.341

Blondeaux, P. \& Seminara, G. 1979 Transizione incipiente al fondo di un'onda di gravitá. Acc. Naz. Lincei, 67, 408-411, (italian)

Blondeaux, P. \& Vittori, G. 1994 Wall imperfections as a triggering mechanism for Stokes-layer transition. J. Fluid Mech., 264, 107-135. 
Blondeaux, P. \& Vittori, G. 1999 Boundary layer and sediment dynamics under sea waves. Adv. Coastal and Ocean Eng., 4, 133-190.

Blondeaux, P. \& Vittori, G. 2011 RANS modelling of the turbulent boundary layer under a solitary wave. Coastal Eng. in press, doi:10.1016/j.coastaleng.2011.07.005.

Bogucki, D.J. \& Redekopp, L.G 1999 A mechanism for sediment resuspension by internal solitary waves. Geophys. Res. Letters, 26 (9), 1317-1320.

Conrad, P.W. \& Criminale, W.O. 1965 The stability of time-dependent laminar flows. Z. Angew. Math. Phys., 16, 233-254.

Costamagna, P., Vittori, G. \& Blondeaux, P. 2003 Coherent structures in oscillatory boundary layers. J. Fluid Mech., 474, 1-33.

Fredsoe, J. \& Deigaard, R. 1992 Mechanics of coastal sediment transport. Adv. Series on Ocean Engineering, 3, World Scientific.

Grimshaw, R. 1971. The solitary wave in water of variable depth. Part 2. J. Fluid Mech., 46 (3), 661-622.

Huerre, P. \& Monkewitz, P.A. 1990. Local and global instabilities in spatially developing flows. Annu. Rev. Fluid Mech., 22, 473-537.

Liu, P.L.F. \& Orfilia, A. 2004 Boundary layer hydrodynamics and bed load sediment transport in oscillating water tunnels. J. Fluid Mech., 520, 83-92.

Liu, P.L.F., Park, Y.S. \& Cowen, E.A. 2007 Boundary layer flow and bed shear stress under a solitary wave. J. Fluid Mech., 574, 449-463.

Mazzuoli, M., Vittori, G. \& Blondeaux, P. 2011 Turbulent spots in oscillatory boundary layers. J. Fluid Mech., 685, 365-376, doi:10.1017/jfm.2011.320.

Mei, C.C. 1989 The applied dynamics of ocean surface waves. Adv. Series on Ocean Eng., 1, World Scientific

Shen, S.F. 1961 Some considerations on the laminar stability of incompressible time-dependent basic flows. J. Aerospace Sci., 28, 397-404 and 417

Sumer, B.M., Jensen P.M., Sorensen, L.B., Fredsoe, J., Liu, P.L.F. \& Carstensen, S. 2010 Coherent structures in wave boundary layers. Part 2. Solitary motion. J. Fluid Mech., 646, 207-231.

Tanaka, H., Sumer, B.M. \& Lodahl, C. 1998 Theoretical and experimental investigation on laminar boundary layers under cnoidal wave motion. Coastal Eng. J., 40 (1), 81-98.

Verzicco, R. \& Vittori, G. 1996 Direct simulation of transition in Stokes layers. Phys. Fluids, 8 (6), 1341-1343.

Vittori, G. 2003 Sediment suspension due to waves. J. Geophys. Res. Oceans, 108 (C6), 3173, 4-1/4-17.

Vittori, G. \& Blondeaux, P. 2008 Turbulent boundary layer under a solitary wave. J. Fluid Mech., 615, 433-443.

Vittori, G. \& Blondeaux, P. 2011 Characterstics of the boundary layer at the bottom of a solitary wave. Coastal Eng., 58 (2), 206-213.

Vittori, G. \& Verzicco, R. 1998 Direct simulation of transition in an oscillatory boundary layer. J. Fluid Mech., 371, 207-232.

Talmon, A.M., Struiksma, N. \& Van Mierlo, M.C.L.M. 1995 Laboratory measurements of the direction of sediment transport on transverse alluvial-bed slopes. J. Hydr. Res., 33, 495-517. 